\title{
Quality of life and nutritional consequences after aboral pouch reconstruction following total gastrectomy for gastric cancer: randomized controlled trial CCG1101
}

\author{
Yuichi Ito $^{1}$ - Takaki Yoshikawa ${ }^{2}$ Michitaka Fujiwara ${ }^{3}$ Hiroshi Kojima ${ }^{4}$. \\ Takanori Matsui ${ }^{4}$ - Yoshinari Mochizuki ${ }^{5}$ Haruhiko Cho $^{2}$ - Toru Aoyama ${ }^{2}$. \\ Seiji Ito ${ }^{1} \cdot$ Kazunari Misawa $^{1} \cdot$ Hiroshi Nakayama $^{6} \cdot$ Yuki Morioka $^{7}$. \\ Akiharu Ishiyama $^{8}$ - Chie Tanaka ${ }^{3}$ - Satoshi Morita ${ }^{9} \cdot$ Junichi Sakamoto ${ }^{10}$. \\ Yasuhiro Kodera ${ }^{3}$
}

Received: 20 May 2015 / Accepted: 28 July 2015/Published online: 14 August 2015

(C) The International Gastric Cancer Association and The Japanese Gastric Cancer Association 2015

\begin{abstract}
Background Total gastrectomy has detrimental effects on postoperative nutritional status and quality of life (QOL), but it is often unavoidable in the treatment of gastric cancer. Roux-en-Y (RY) is the most common reconstruction method following total gastrectomy. Trials to explore other means of reconstruction have been conducted but have failed to identify a method that is globally accepted. Methods Aboral pouch reconstruction (AP), in which an anisoperistaltic jejunal pouch is created in the Y limb of the $\mathrm{RY}$ reconstruction, is considered effective and technically feasible. A prospective randomized trial was conducted to
\end{abstract}

Yasuhiro Kodera

ykodera@med.nagoya-u.ac.jp

1 Department of Gastroenterological Surgery, Aichi Cancer Center Chuo Hospital, Nagoya, Japan

2 Department of Gastrointestinal Surgery, Kanagawa Cancer Center, Yokohama, Japan

3 Department of Gastroenterological Surgery, Nagoya University Graduate School of Medicine, Nagoya, Japan

4 Department of Gastroenterological Surgery, Aichi Cancer Center Aichi Hospital, Okazaki, Japan

5 Department of Surgery, Komaki Municipal Hospital, Komaki, Japan

6 Department of Surgery, Nagoya Medical Center, Nagoya, Japan

7 Department of Surgery, Ichinomiya Municipal Hospital, Ichinomiya, Japan

8 Department of Surgery, Okazaki City Hospital, Okazaki, Japan

9 Department of Biomedical Statistics and Bioinformatics, Kyoto University Graduate School of Medicine, Kyoto, Japan

10 Tokai Central Hospital, Kagamigahara, Japan compare AP with RY. Gastric cancer patients requiring total gastrectomy for R0 resection were randomly assigned during surgery to receive either RY $(n=51)$ or AP $(n=49)$. Postoperative QOL as assessed by the EORTC QLQ-C30 and STO22, body composition, and morbidity were compared between the two reconstruction methods. The physical functioning score of the QLQ-C30 was selected as the primary endpoint.

Results The incidences of postoperative complications were similar between the two groups ( $29 \%$ in the RY group and $27 \%$ in the AP group). No significant difference was observed in the physical functioning score, and the superiority of AP was demonstrated only for the nausea and vomiting score at 12 months $(p=0.041)$ and the reflux score at 1 month $(p=0.036)$. No significant differences were observed in body composition or serum biochemistry.

Conclusions Although AP was safely implemented, no increased benefits in nutritional or QOL-related parameters were observed for this method over RY within 12 months postoperatively.

Keywords Total gastrectomy $\cdot$ Reconstruction $\cdot$ Patientreported outcome $\cdot$ Jejunal pouch $\cdot$ Body composition

\section{Introduction}

According to GLOBOCAN 2012 (http://globocan.iarc.fr), gastric cancer is the third most common cause of cancer deaths worldwide, resulting in 723,000 deaths in 2012. Surgery remains the most essential part of multimodal therapy for patients with this type of cancer. Total gastrectomy is mandatory for curative resection of cancer that 
involves the upper part of the stomach, despite its detrimental effects on health and quality of life (QOL). Rouxen-Y (RY), the most common type of reconstruction performed after total gastrectomy, is simple, safe, and associated with a low incidence of reflux symptoms [1]. However, this method often fails to prevent various other postgastrectomy symptoms that adversely affect the QOL of patients, and eventually leads to malnutrition. Numerous other reconstruction methods have been proposed and explored, but the optimal method that is clinically beneficial, technically simple, and cost-effective remains elusive.

Several studies have evaluated the effect of creating a jejunal pouch to compensate for the loss of reservoir capacity [2-9]. The construction of a pouch was once considered complex and time-consuming, but these disadvantages are no longer a concern in the era of mechanical staplers. However, pouch reconstruction is sometimes associated with food stasis and excessive pouch dilatation, and the optimal size and site of the pouch remain a source of controversy. More recently, aboral pouch reconstruction (AP), in which an anisoperistaltic jejunal pouch is created in the $Y$ limb of the RY reconstruction [10], has emerged as a relatively inexpensive option that is feasible, even using a laparoscopic approach. In addition, this is the only pouchreconstruction method that can be reasonably employed after transhiatal extended gastrectomy in junctional tumors. In this method, the pouch not only endows reservoir capacity but also allows food and digestive fluids from the Roux limb to mix well, thereby theoretically enhancing digestion and nutrient absorption. In a randomized trial with RY as a control arm, AP was found to be superior in terms of postoperative serum cholesterol levels, lipid absorption, and QOL [10]. However, this study was a small single-institutional attempt with no predetermined endpoints or sample size calculations based on statistical considerations.

Therefore, we conducted a prospective randomized controlled trial comparing AP and RY, in which the postoperative physical functioning scores on the European Organization for Research and Treatment of Cancer (EORTC) Quality of Life Questionnaire-Core 30 (QLQC30), an established instrument for the assessment of QOL [11], were evaluated as the primary endpoint. The secondary endpoints included other QLQ-C30 subscales, body composition parameters, and safety.

\section{Methods}

\section{Patient eligibility}

CCOG1101, a multi-institutional prospective randomized trial, was designed to test the hypothesis that AP results in a superior physical functioning score. Patients were randomized during surgery to receive either $\mathrm{RY}$ or $\mathrm{AP}$ after total gastrectomy for gastric cancer. Comparisons of patient-reported outcomes, body composition, nutritional status, and safety were made between the two types of reconstructions.

The eligibility criteria for preoperative registration were as follows: (1) histologically proven gastric adenocarcinoma; (2) total gastrectomy required for R0 resection; (3) performance status of 0 or 1 ; (4) age of between 20 and 80 years; (5) esophageal invasion of $<3 \mathrm{~cm}$ and no need for thoracotomy; (6) no history of upper abdominal surgery with the exception of laparoscopic cholecystectomy; (7) no pretreatment with chemotherapy or chemoradiation; (8) the ability to understand and respond to the questionnaires; (9) no severe organ malfunctions that excluded the patient from being indicated for standard surgical treatment; and (10) the provision of written informed consent. The exclusion criteria were as follows: (1) cancer of the gastric remnant; (2) linitis plastica type gastric cancer; (3) the need for co-resection of other organs with the exception of cholecystectomy; (4) currently under treatment or being followed up for other malignancies; (5) body mass index of $>28 \mathrm{~kg} / \mathrm{m}^{2}$; (6) uncontrollable hypertension or diabetes mellitus; and (7) regular steroid use.

The patients were registered by fax or telephone for consideration as candidates for the trial and were randomized during surgery after the surgical team confirmed that the following criteria were fulfilled: (1) macroscopic diagnosis of up to T4a, N2 and M0; (2) total gastrectomy required for curative resection with adequate surgical margins; and (3) no need for thoracotomy. Randomization was performed by the minimization method and was stratified by center, intraoperative estimation of tumor depth (T1a and T1b/T2 and T3/T4a), and surgical approach (open/laparoscopic). The results were conveyed to the surgeons either by fax or telephone.

The patients were asked to complete the EORTC QLQ$\mathrm{C} 30$ and stomach module (STO22) questionnaires before surgery and at 1 month, 6 months, and 12 months after surgery. Additionally, they underwent blood tests and measurements of body composition by the bioimpedance method using a TANITA DC-320 body analyzer (TANITA Corp., Tokyo, Japan) at the baseline, 1 month, 6 months, and 12 months after surgery. Follow-up evaluation either by abdominal computed tomography or abdominal ultrasound was conducted every 6 months.

All patients were informed of the nature of the study and provided written consent. The study was approved by the Institutional Review Boards of all 12 participating institutions. The trial was registered in the University Hospital Medical Information Network's Clinical Trials Registry (UMIN-CTR; number 000006155). 


\section{Surgical treatment and postoperative adjuvant chemotherapy}

Standard surgery was performed for all patients in accordance with the Japanese Gastric Cancer Treatment Guidelines [12], except that the laparoscopic approach (which is not considered a standard in the guidelines) was allowed for the patients with a preoperative diagnosis of stage I cancer. Figure 1 summarizes the characteristics of each method of reconstruction. The $\mathrm{Y}$ limb in RY was constructed by hand sewing, or by using a linear stapler where the length of anastomosis had to be less than $4 \mathrm{~cm}$. The Y limb in AP was created by anisoperistaltic side-toside anastomosis using two $75-\mathrm{mm}$ linear staplers. In addition to organizing a series of meetings among the participants of the trial to discuss the surgical techniques and study design, a video of an AP reconstruction exemplarily conducted by one of the participants (Y. I.) was distributed to ensure that the procedure would be performed identically by all of the participants. The decision of whether to use the antecolic or retrocolic route was left to the preference of each surgeon.

D2 lymph node dissection conforming to the Japanese Gastric Cancer Treatment Guidelines was performed for the patients with a preoperative and intraoperative diagnosis of T2-T4a gastric cancer, while those with T1 disease received a $\mathrm{D} 1+$ dissection. After pathological examination of the resected specimens, the patients with confirmed stage II-III disease were treated with postoperative S-1 monotherapy for 12 months.

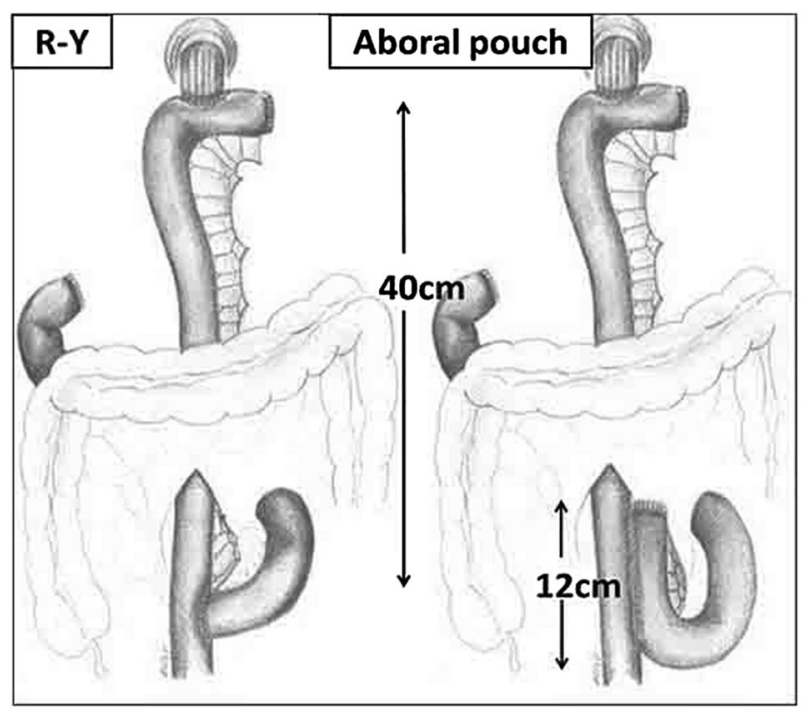

Fig. 1 The Y limb in the Roux-Y group was constructed either by hand sewing or using a linear stapler where the length of anastomosis had to be less than $4 \mathrm{~cm}$. The $\mathrm{Y}$ limb in the aboral pouch group was created by anisoperistaltic side-to-side anastomosis using two $75-\mathrm{mm}$ linear staplers

\section{Assessment of QOL}

The EORTC QLQ-C30 questionnaire is composed of 30 items for assessing the QOL of cancer patients. These items are categorized into the global health status/QOL scale, 5 functioning scales (physical, role, cognitive, emotional, and social), 3 symptom scales (fatigue, pain, nausea and vomiting), and 6 single items (dyspnea, sleep disturbance, appetite loss, constipation, diarrhea, and financial difficulties). The EORTC STO22 is a questionnaire tailored to gastric cancer patients that is composed of 22 items [13, 14]. The 22 items are categorized into 5 symptom scales (dysphagia, abdominal pain, reflux, eating restriction, and anxiety) and 4 single items (dry mouth, taste problems, body image, and hair loss). Missing data were processed according to the EORTC scoring manuals [15], and all scales were linearly transformed to scores of 0 to 100 . Consequently, high scores denoted favorable outcomes regarding global health status and the functioning scales, whereas low scores on the symptom scales and items indicated favorable outcomes.

In the current study, the validated Japanese language versions of the EORTC QLQ-C30 and STO22 were used to assess the patient-reported outcomes [16, 17]. The patients were provided the questionnaire sheets by their attending physician and were asked to complete them prior to surgery and to send them by mail to the independent data center run by the Chubu Clinical Oncology Group (CCOG). Further questionnaire sheets were sent directly from the data center to the patients at one, six, and twelve months after surgery to be completed and returned by mail.

\section{Sample size calculation and statistical considerations}

The primary endpoint was the physical functioning score, as measured with the EORTC QLQ-C30 at 12 months after surgery. The secondary endpoints were the other subscales of the QLQ-C30 and STO22, body composition, nutritional status as evaluated by blood testing, operation time, blood loss during surgery, and morbidity. An intergroup difference of 10 points in the QLQ-C30 physical functioning score obtained at 12 months after surgery was expected out of a scale of 100. Assuming a common standard deviation of 15, 37 patients were required per group for analysis given an alpha error on both sides of $5 \%$ and a detectability of $80 \%$. A sample size of 90 patients was used, taking into consideration missing QOL survey data and the emergence of ineligible patients for other reasons. The chi-squared test was used to compare categorical data, and Student's $t$ test was used for continuous variables. A $p$ value of less than 0.05 was considered significant. The JMP statistical package, version 10 (SAS, Cary, NC, USA), was used for statistical analysis. 


\section{Results}

\section{Patient demographics}

A total of 121 patients were registered preoperatively from September 2011 to November 2012. Of them, 101 were deemed eligible during surgery and were enrolled and randomly allocated to either of the treatment arms. Peritoneal deposits were found in one patient after being randomized and allocated to the RY arm. This patient was subsequently considered unresectable. Thus, 100 patients were eligible for comparison, of which 51 were allocated to the RY group and 49 to the AP group (Fig. 2). The patient demographics are presented in Table 1. No significant differences were observed between the two groups in terms of age, gender, preoperative weight, preoperative body mass index, tumor diameter, depth of invasion, or stage distribution.

\section{Safety analysis}

Short-term outcomes stratified by the operative procedure are presented in Table 2. No significant differences were observed between the two groups in terms of operation time, blood loss, postoperative hospital stay, or morbidity. No in-hospital mortality was observed. Postoperative complications of grade 2 or higher according to CTCAE ver. 4.0 are shown in Table 2 . There were no surgical complications that were considered to be specific to AP. Seventeen (33\%) patients in the RY group and 15 (31\%) patients in the AP group underwent postoperative adjuvant chemotherapy according to the Japanese Gastric Cancer Treatment Guidelines. Recurrence was observed within 1 year in $5(9.8 \%)$ patients in the RY group and $4(8.2 \%)$ patients in the AP group.

\section{Patient-reported outcomes}

The questionnaires were completed and returned by 91,94 , 93 , and $88 \%$ of the patients at the baseline and at 1 month, 6 months, and 12 months postoperatively. The scores for the global health status and the five functioning scales decreased in both groups at 1 month after surgery and then gradually recovered to the baseline level at 12 months postoperatively (Fig. 3). No significant difference was observed in the physical functioning score (the primary endpoint) at 12 months postoperatively. Among the QLQC30 and STO 22 symptom scores, the nausea and vomiting score showed significant improvement in the AP group $(p=0.041)$ at 1 year after surgery. The reflux score was significantly better in the AP group at 1 month after surgery $(p=0.036)$. Additionally, the scores for abdominal pain, dry mouth, taste problems, and body image showed improvements in the AP group, although these differences did not reach statistical significance (Fig. 4).

\section{Body composition and nutritional consequences}

Changes in serum nutritional and body composition parameters in both groups are presented in Table 3. Nutritional status was evaluated by blood testing, but no significant difference was noted between the two groups. The body weight loss at 12 months after surgery was approximately $9 \mathrm{~kg}$ for both reconstruction methods. No significant differences were observed between the two groups in total body weight, fat mass, or lean body mass.

\section{Discussion}

Various reconstruction methods after total gastrectomy have been reported, but no consensus has been reached regarding a standard method. Due to the prolongation of survival through early diagnosis and the need for tolerance to evidence-based postoperative adjuvant therapies for gastric cancer that have been reported in these patients [18, 19], a reconstruction method that adequately maintains their nutritional status and QOL after gastrectomy has become a matter of paramount importance. Consequently, various trials examining jejunal pouch reconstruction, mainly at the esophagojejunal anastomosis, as a means of maintaining reservoir capacity after total gastrectomy have been conducted. Gertler et al. conducted meta-analysis, concluding that pouch reconstruction mainly at the esophagojejunal anastomosis may offer advantages [20], but no definitive conclusion has been reached due to the small size of each of the trials.

The current randomized trial was conducted in a multiinstitutional setting because all of the surgeons at the participating institutions considered RY to be a tentative standard due to the lack of evidence for selecting more complex procedures; however, an agreement was reached on the theoretical advantage of AP and its technical simplicity compared with other types of pouch reconstruction. This allowed for the enrollment of a generous number of patients compared with the previous trials conducted during the 2 years of patient accrual, and the safety and feasibility of AP was demonstrated despite the fact that most of the surgeons lacked a training period. The AP technique using linear staplers was found to be easily performed by surgeons with sufficient experience in gastric cancer surgery.

However, differences in the physical functioning scores (the primary endpoint) were disappointingly scarce. The QLQ-C30 and STO22 were employed in the current study 


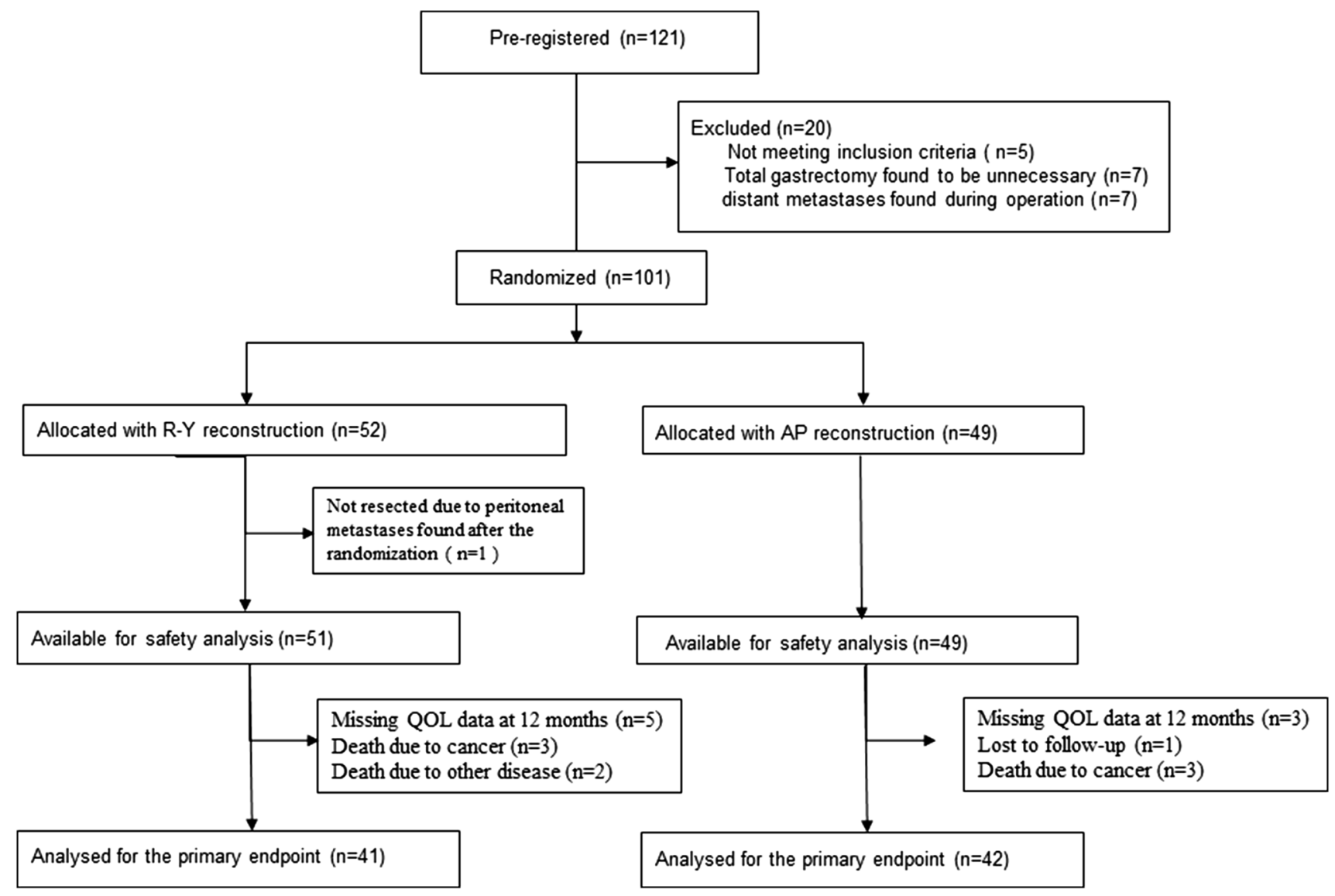

Fig. 2 CONSORT flow diagram. Of 101 randomized patients, 52 were allocated to receive Roux-en-Y reconstruction and 49 to undergo aboral pouch reconstruction. The numbers of patients available for analysis of the primary endpoint were 41 and 42 in each group, respectively

Table 1 Patient demographics

\begin{tabular}{llll}
\hline & R-Y group $(n=51)$ & AP group $(n=49)$ & $p$ value \\
\hline Age & $65.3 \pm 8.7^{*}$ & $66.9 \pm 7.6^{*}$ & 0.343 \\
Sex $(\mathrm{M} / \mathrm{F})$ & $37 / 14$ & $34 / 15$ & 0.728 \\
Preoperative body weight $(\mathrm{kg})$ & $60.1 \pm 10.6^{*}$ & $58.4 \pm 10.0^{*}$ & 0.401 \\
Preoperative BMI $\left(\mathrm{kg} / \mathrm{m}^{2}\right)$ & $22.5 \pm 2.9^{*}$ & $22.3 \pm 2.2^{*}$ & 0.669 \\
Tumor diameter $(\mathrm{cm})$ & $4.4 \pm 3.3^{*}$ & $4.0 \pm 2.2^{*}$ & 0.563 \\
Tumor depth $(\mathrm{T} 1 / \mathrm{T} 2 / \mathrm{T} 3 / \mathrm{T} 4)$ & $27 / 8 / 5 / 11$ & $24 / 4 / 13 / 8$ & 0.139 \\
Stage $(\mathrm{I} / \mathrm{II} / \mathrm{III} / \mathrm{IV})$ & $28 / 12 / 9 / 2$ & $27 / 8 / 12 / 2$ & 0.751 \\
\hline
\end{tabular}

$*$ Mean $\pm \mathrm{SD}$

because they have been established as globally applicable instruments for measuring patient-reported outcomes. We previously performed a pilot study comparing postoperative QOL among patients treated by open total gastrectomy with RY, open distal gastrectomy, and laparoscopy-assisted distal gastrectomy [21]. The physical functioning score at 12 months postoperatively was found to be outstandingly poor in that study after total gastrectomy compared with distal gastrectomy, and this finding led to its selection as a primary endpoint in the current study. However, our results indicated that the differences in this parameter as a consequence of adding a jejunal pouch (if any) should be considered trivial compared with those caused by the presence or absence of the remnant stomach. Additionally, the sample size of the trial was insufficient to detect significant differences in favor of AP for a majority of the items included in the QLQ-C30 and STO22, with the exception of the incidence of reflux at 1 month and nausea and vomiting at 12 months after surgery. Since these items were not the primary endpoint basis for the calculation of the sample size of the study, these consolatory results will have to be interpreted rather sparingly at this time. There 
Table 2 Type of surgery and short-term outcome of the patients enrolled
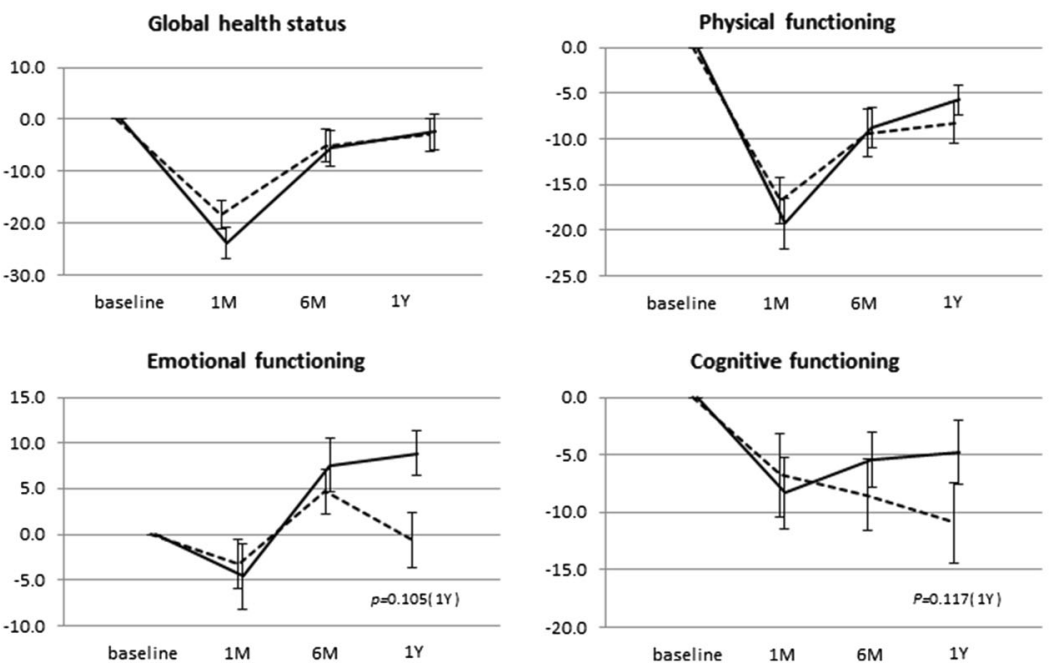

\begin{tabular}{|c|c|c|c|}
\hline & $\mathrm{R}-\mathrm{Y}$ group $(n=51)$ & AP group $(n=49)$ & $p$ value \\
\hline OpenTG/laparoscopic TG & $35 / 16$ & $33 / 16$ & 0.89 \\
\hline Conversion to OTG from LTG & 1 & 1 & 1 \\
\hline LN dissection (D1/D2) & $30 / 21$ & $26 / 23$ & 0.56 \\
\hline Splenectomy (yes/no) & $18 / 33$ & $19 / 30$ & 0.72 \\
\hline Operation time (min) & $293 \pm 64^{*}$ & $287 \pm 84^{*}$ & 0.7 \\
\hline Blood loss (ml) & $322 \pm 288^{*}$ & $274 \pm 267 *$ & 0.39 \\
\hline Blood transfusion & 2 & 1 & 0.58 \\
\hline Duration of hospitalization & $13(7-158)^{* *}$ & $12(8-165)^{* *}$ & 0.92 \\
\hline Morbidity (all grades, \%) & $15(29 \%)$ & $13(27 \%)$ & 0.75 \\
\hline Morbidity $\geq \mathrm{G} 2(\%)$ & $11(22 \%)$ & $9(18 \%)$ & 0.69 \\
\hline Anastomotic leakage & 4 & 1 & 0.18 \\
\hline Pancreatic fistula & 3 & 4 & 0.66 \\
\hline Biliary fistula & 0 & 1 & 0.31 \\
\hline Abdominal abscess & 2 & 4 & 0.37 \\
\hline Mediastinal abscess & 1 & 0 & 0.33 \\
\hline Pleural effusion & 2 & 0 & 0.16 \\
\hline Postoperative hemorrhage & 1 & 1 & 0.98 \\
\hline Stomal stenosis & 2 & 0 & 0.16 \\
\hline Ileus & 1 & 0 & 0.33 \\
\hline Anorexia & 1 & 1 & 0.98 \\
\hline Reflux esophagitis & 0 & 1 & 0.31 \\
\hline Mortality & 0 & 0 & 1 \\
\hline
\end{tabular}

$*$ Mean $\pm \mathrm{SD}$, ** median (range)
Fig. 3 Global health status and functioning scores determined using the EORTC QLQ-C30 and stratified by the method of reconstruction. The scores were generally the lowest at 1 month postoperatively but recovered to the baseline by 12 months after surgery. No significant

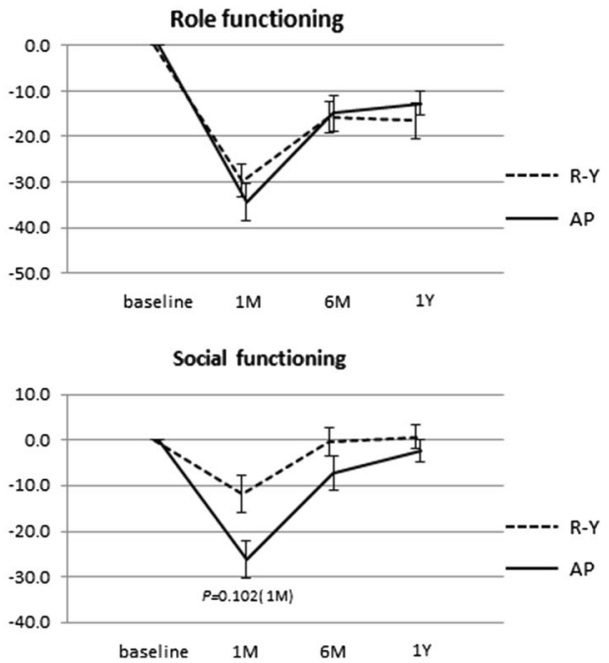

differences were observed between the groups in any of the functioning scores at any of the time points, including the physical functioning score at 12 months, which was the primary endpoint

indicators, including body weight, lean body mass, and nutritional status, as evaluated by blood testing. Overall, this trial did not robustly demonstrate a superiority of AP reconstruction over RY reconstruction. 

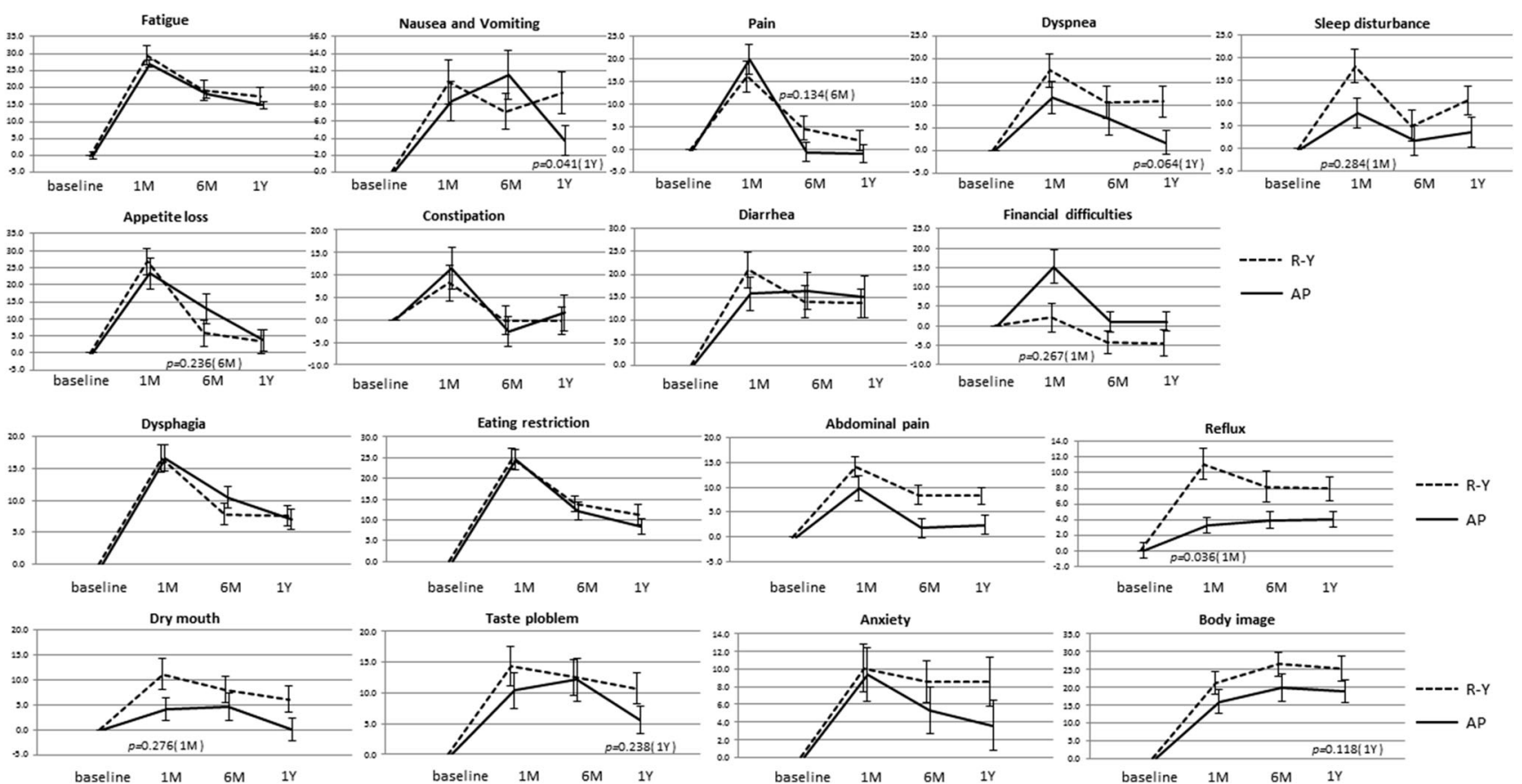

Fig. 4 Symptom scores determined using the EORTC QLQ-C30 and STO22 and stratified by the method of reconstruction. The scores were the lowest at 1 month postoperatively and often had not

recovered to the baseline at 12 months after surgery. Several of the symptom scores tended to be lower for the aboral pouch group, but the majority of these differences were not statistically significant

Table 3 Comparison of changes in serum nutritional and body composition parameters between the R-Y group and the AP group

\begin{tabular}{|c|c|c|c|c|c|c|c|c|}
\hline & \multicolumn{4}{|c|}{ R-Y group $(n=51)$} & \multicolumn{4}{|c|}{ AP group $(n=49)$} \\
\hline & Baseline & $1 \mathrm{M}$ & $6 \mathrm{M}$ & $12 \mathrm{M}$ & Baseline & $1 \mathrm{M}$ & $6 \mathrm{M}$ & $12 \mathrm{M}$ \\
\hline Total protein $(\mathrm{g} / \mathrm{dl})$ & $7.00 \pm 0.46$ & $6.81 \pm 0.47$ & $6.76 \pm 0.49$ & $6.87 \pm 0.49$ & $7.02 \pm 0.48$ & $6.67 \pm 0.43$ & $6.83 \pm 0.42$ & $6.90 \pm 0.33$ \\
\hline Albumin (g/dl) & $4.18 \pm 0.35$ & $3.86 \pm 0.43$ & $4.01 \pm 0.37$ & $4.03 \pm 0.31$ & $4.26 \pm 0.38$ & $3.87 \pm 0.43$ & $4.09 \pm .40$ & $4.13 \pm 0.26$ \\
\hline $\begin{array}{l}\text { Total cholesterol } \\
(\mathrm{mg} / \mathrm{dl})\end{array}$ & $199 \pm 35.7$ & $167 \pm 25.0$ & $174 \pm 31.3$ & $163 \pm 24.2$ & $187 \pm 31.1$ & $163 \pm 34.6$ & $169 \pm 32.5$ & $169 \pm 39.3$ \\
\hline Triglyceride $(\mathrm{mg} / \mathrm{dl})$ & $159 \pm 17.7$ & $104 \pm 46.1$ & $87.1 \pm 39.0$ & $33.2 \pm 33.1$ & $147 \pm 17.2$ & $98 \pm 27.9$ & $86.3 \pm 29.4$ & $91.5 \pm 38.7$ \\
\hline Calcium (mg/dl) & $9.34 \pm 0.41$ & $9.27 \pm 0.46$ & $9.27 \pm 0.46$ & $9.17 \pm 0.31$ & $9.35 \pm 0.54$ & $9.29 \pm 0.53$ & $9.33 \pm 0.43$ & $9.32 \pm 0.39$ \\
\hline Iron (ug/dl) & $78.8 \pm 41.2$ & $57.0 \pm 27.4$ & $89.8 \pm 30.3$ & $99.3 \pm 38.0$ & $79.6 \pm 39.2$ & $54.4 \pm 21.6$ & $91.0 \pm 26.1$ & $95.9 \pm 35.5$ \\
\hline $\begin{array}{l}\text { Lymphocyte count (/ } \\
\text { mmJ) }\end{array}$ & $1635 \pm 642$ & $1659 \pm 764$ & $1680 \pm 848$ & $1639 \pm 750$ & $1648 \pm 700$ & $1661 \pm 694$ & $1763 \pm 893$ & $1869 \pm 762$ \\
\hline Hemoglobin (g/dl) & $13.2 \pm 1.82$ & $12.1 \pm 1.41$ & $12.2 \pm 1.69$ & $12.2 \pm 1.52$ & $13.4 \pm 1.50$ & $11.9 \pm 1.07$ & $12.1 \pm 1.51$ & $12.1 \pm 1.51$ \\
\hline Body weight (kg) & $59.1 \pm 11.1$ & $53.4 \pm 9.70$ & $51.6 \pm 8.94$ & $50.5 \pm 8.56$ & $57.2 \pm 9.65$ & $51.5 \pm 3.50$ & $4 \mathrm{~S} .7 \pm \mathrm{S} .96$ & $48.5 \pm 9.62$ \\
\hline Fat $(\mathrm{kg})$ & $13.8 \pm 4.58$ & $10.7 \pm 4.77$ & $8.45 \pm 3.97$ & $7.54 \pm 3.42$ & $12.4 \pm 4.02$ & $9.78 \pm 3.89$ & $7.14 \pm 3.76$ & $7.06 \pm 3.68$ \\
\hline Muscle $(\mathrm{kg})$ & $43.2 \pm 7.68$ & $40.1 \pm 7.44$ & $41.5 \pm 6.58$ & $40.3 \pm 6.74$ & $42.3 \pm 8.06$ & $39.6 \pm 7.18$ & $39.5 \pm 6 . S 6$ & $39.3 \pm 7.51$ \\
\hline
\end{tabular}

In the original trial that showed a superiority of AP in terms of some nutritional parameters, Horvath et al. created pouches with lengths of $15 \mathrm{~cm}$ for AP [10]; in contrast, the pouches constructed in the present study had a median length of $12 \mathrm{~cm}$ (range: $8-15 \mathrm{~cm}$ ) due to the regulation that two 75-mm linear staplers should be used to minimize cost. In addition, the authors sought advice from investigators who are enthusiastic advocates of pouch reconstruction both at the esophagojejeunal anastomosis [22] and in the Y limb [23], and were warned that excessive pouch dilatation, one of the adverse events associated with pouch reconstruction, is more likely to occur in patients with large pouches. Although the optimal jejunal pouch length for AP remains to be elucidated, we considered it unlikely that the patient-reported outcomes in the current study could have been influenced by the subtle differences in the capacities 
of the reservoirs when compared with the original report of a case series involving more heavily built Caucasian patients [10].

As a result of a pivotal phase III trial [18], postoperative adjuvant chemotherapy has become the standard therapy for gastric cancer patients who have undergone stage II/III curative resection in Japan. Consequently, S-1 adjuvant chemotherapy was performed for 12 months for more than $30 \%$ of the patients in both groups. Chemotherapy may have affected the QOL of these subgroups and nullified the benefits of AP. However, no significant differences in the various relevant parameters were observed, even when analysis was limited to the patients who had not undergone postoperative adjuvant chemotherapy (data not shown). Additionally, the investigators had originally expected that this subset would actually benefit from AP through improved nutritional status, which would minimize the adverse effects of chemotherapy [19].

The combination of the QLQ-C30 with the STO22 was used to assess the patient-reported outcomes among gastric cancer patients who had undergone a wide array of anticancer therapies, and was not specifically compiled to address the issue of postgastrectomy symptoms. Several instruments that more specifically address patient-reported outcomes following gastrectomy are now available, albeit in Japanese [23, 24], and studies using these instruments that have shown some noteworthy results are now starting to be published [25-27]. Furthermore, pouch reconstruction in general has been proposed to improve long-term QOL when evaluated after as long as 30 months postsurgery [1]. This finding suggests that the 12-month observation period used in the current study may have been too short. Further comparison after a longer follow-up period and the use of another questionnaire with a greater focus on postgastrectomy symptoms [23] may reveal some advantages or disadvantages of AP. A protocol amendment in this regard is currently in progress, and future analysis based on the results of this study might generate some new findings.

\section{Conclusion}

AP after total gastrectomy is safe and feasible. However, the benefit of this method over the conventional RY method in terms of QOL and nutritional status was not shown as of 12 months postoperatively in this randomized trial.

Acknowledgments We thank all of the patients and the following investigators who also participated in the CCOG1101 study in addition to the authors: Kiyoshi Ishigure, Konan Kosei Hospital, Konan, Aichi; Katsumi Koshikawa, Nagoya Memorial Hospital, Nagoya, Aichi; Takashi Kinoshita, Yokkaichi Municipal Hospital, Yokkaichi,
Mie; Shinichi Fujitake, Gamagori City Hospital, Gamagori, Aichi, Japan. This study was supported in part by the Japanese Ministry of Education and Science and the non-profit organization CCOG.

\section{Compliance with ethical standards}

Conflict of interest Dr. Yoshikawa reports grants and personal fees from Covidien and personal fees from Johnson \& Johnson outside the submitted work. Dr. Kodera reports grants and personal fees from Johnson \& Johnson, grants and personal fees from Covidien, and personal fees from Hogy Medical outside the submitted work.

Statement of human rights All procedures followed were in accordance with the ethical standards of the responsive committee on human experimentation (institutional and national) and with the Declaration of Helsinki (1964 and later versions).

Informed consent Informed consent (or a substitute for it) to being included in the study was obtained from all patients who took part in the study.

\section{References}

1. Fein M, Fuchs KH, Thalheimer A, Freys SM, Heimbucher J, Thiede A. Long-term benefits of Roux-en-Y pouch reconstruction after total gastrectomy: a randomized trial. Ann Surg. 2008;247:759-65.

2. Troidl H, Kusche J, Vestweber KH, Eypasch E, Maul U. Pouch versus esophagojejunostomy after total gastrectomy: a randomized clinical trial. World J Surg. 1987;11:699-712.

3. Nakane Y, Okumura S, Akehira K, Okamura S, Boku T, Okusa T, et al. Jejunal pouch reconstruction after total gastrectomy for cancer. A randomized controlled trial. Ann Surg. 1995;222:27-35.

4. Bozzetti F, Bonfanti G, Castellani R, Maffioli L, Rubino A, Diazzi G, et al. Comparing reconstruction with Roux-en-Y to a pouch following total gastrectomy. J Am Coll Surg. 1996;183:243-8.

5. Schwarz A, Buchler M, Usinger K, Rieger H, Glasbrenner B, Friess $\mathrm{H}$, et al. Importance of the duodenal passage and pouch volume after total gastrectomy and reconstruction with the Ulm pouch: prospective randomized clinical study. World J Surg. 1996;20:60-6.

6. Liedman B, Andersson H, Bosaeus I, Bosaeus I, Hugosson I, Lundell L. Changes in body composition after gastrectomy: results of a controlled, prospective clinical trial. World J Surg. 1997;21:416-20.

7. Iivonen MK, Koskinen MO, Ikonen TJ, Matikainen MJ. Emptying of the jejunal pouch and Roux-en-Y limb after total gastrectomy - a randomised, prospective study. Eur J Surg. 1999;165:742-7.

8. Adachi S, Inagawa S, Enomoto T, Shinozaki E, Oda T, Kawamoto T. Subjective and functional results after total gastrectomy: prospective study for longterm comparison of reconstruction procedures. Gastric Cancer. 2003;6:24-9.

9. Kono K, Iizuka H, Sekikawa T, Sugai H, Takahashi A, Fujii H, et al. Improved quality of life with jejunal pouch reconstruction after total gastrectomy. Am J Surg. 2003;185:150-4.

10. Horvath OP, Kalmar K, Cseke L, Poto L, Zambo K. Nutritional and life-quality consequences of aboral pouch construction after total gastrectomy: a randomized, controlled study. Eur J Surg Oncol. 2001;27:558-63.

11. Aaronson NK, Ahmedzai S, Bergman B, Bullinger M, Cull A, Duez NJ, et al. The European Organization for Research and 
Treatment of Cancer QLQ-C30: a quality-of-life instrument for use in international clinical trials in oncology. J Natl Cancer Inst. 1993;85:365-76.

12. Japanese Gastric Cancer Association. Japanese Gastric Cancer Treatment Guidelines 2010 (ver. 3). Gastric Cancer. 2011;14:113-23.

13. Vickery CW, Blazeby JM, Conroy T, Arraras J, Sezer O, Koller $\mathrm{M}$, et al. Development of an EORTC disease-specific quality of life module for use in patients with gastric cancer. Eur J Cancer. 2001;37:966-71.

14. Blazeby JM, Conroy T, Bottomley A, Vickery C, Arraras J, Sezer $\mathrm{O}$, et al. Clinical and psychometric validation of a questionnaire module, the EORTC QLQ-STO 22, to assess quality of life in patients with gastric cancer. Eur J Cancer. 2004;40:2260-8.

15. Fayers PM, Aaronson NK, Bjordal K et al. EORTC QLQ-C30 scoring manual. 3rd ed. Brussels: EORTC; 2001.

16. Kobayashi K, Takeda F, Teramukai S, Gotoh I, Sakai H, Yoneda $\mathrm{S}$, et al. A cross-validation of the European Organization for Research and Treatment of Cancer QLQ-C30 (EORTC QLQC30) for Japanese with lung cancer. Eur J Cancer. 1998;34:810-5.

17. Morita S, Kaptein AA, Oba K, Sakamoto J. The domain structure of the EORTC QLQ-STO22 supported by Japanese validation data. Psychooncology. 2008;17:474-9.

18. Sakuramoto S, Sasako M, Yamaguchi T, Kinoshita T, Fujii M, Nashimoto A, et al. Adjuvant chemotherapy for gastric cancer with S-1, an oral fluoropyrimidine. $\mathrm{N}$ Engl $\mathrm{J}$ Med. 2007;357:1810-20.

19. Aoyama T, Kawabe T, Fujikawa H, Hayashi T, Yamada T, Tsuchida K, et al. Loss of lean body mass as an independent risk factor for continuation of S-1 adjuvant chemotherapy for gastric cancer. Ann Surg Oncol. 2015;22:2560-6.

20. Gertler R, Rosenberg R, Feith M, Schuster T, Friess H. Pouch vs. no pouch following total gastrectomy: meta-analysis and systematic review. Am J Gastroenterol. 2009;104:2838-51.
21. Kobayashi D, Kodera Y, Fujiwara M, Koike M, Nakayama G, Nakao A. Assessment of quality of life after gastrectomy using EORTC QLQ-C30 and STO22. World J Surg. 2011;35:357-64.

22. Ikeda M, Ueda T, Shiba T. Reconstruction after total gastrectomy by the interposition of a doble jejunal pouch using a double stapling technique. Br J Surg. 1998;85:398-402.

23. Nakada $K$, Ikeda $M$, Takahashi $M$, Kinami S, Yoshida M, Uenosono $\mathrm{Y}$, et al. Characteristics and clinical relevance of postgastrectomy syndrome assessment scale (PGSAS)-45: newly developed integrated questionnaires for assessment of living status and quality of life in postgastrectomy patients. Gastric Cancer. 2015;18:147-58.

24. Nakamura M, Hosoya Y, Yano M, Doki Y, Miyashiro I, Kurashina $\mathrm{K}$, et al. Extent of gastric resection impacts patient quality of life: the dysfunction after upper gastrointestinal surgery for cancer (DAUGS32) scoring system. Ann Surg Oncol. 2011;18:314-20.

25. Namikawa $\mathrm{T}$, Hiki $\mathrm{N}$, Kinami $\mathrm{S}$, Okabe $\mathrm{H}$, Urushihara $\mathrm{T}$, Kawahira $\mathrm{H}$, et al. Factors that minimize postogastrectomy symptoms following pylorus-preserving gastrectomy: assessment using a newly developed scale (PGSAS-45). Gastric Cancer. 2015;18:397-406.

26. Terashima M, Tanabe K, Yoshida M, Kawahira H, Inada T, Okabe $\mathrm{H}$, et al. Postgastrectomy syndrome assessment scale (PGSAS)-45 and changes in body weight are useful tools for evaluation of reconstruction methods following distal gastrectomy. Ann Surg Oncol. 2014;21(Suppl 3):370-8.

27. Takiguchi $N$, Takahashi $M$, Ikeda $M$, Inagawa $S$, Ueda $S$, Nobuoka T, et al. Long-term quality-of-life comparison of total gastrectomy and proximal gastrectomy by postgastrectomy syndrome assessment scale (PGSAS-45): a nationwide multi-institutional study. Gastric Cancer. 2015;18:407-16. 